\title{
Descentralización del Sector Salud en México: el Caso de Baja California
}

\author{
Decentralising the Health Sector in Baja California, Mexico
}

Martha C. Jaramillo-Cardona

Universidad Autónoma de Baja California, México. martha_06@uabc.mx

Recibido 23 Abril 2007/Enviado para Modificación 9 Septiembre 2007/Aceptado 2 Octubre 2007

\section{RESUMEN}

Objetivo Caracterizar y analizar las diferentes actuaciones del gobierno estatal para implementar la política de descentralización de los servicios públicos de salud desde 1996 hasta el año 2004, en el Estado de Baja California.

Métodos Estudio comparativo con información secundaria que incluye los años 1996 y 2004 . Se analizaron cuatro categorías centrales: disponibilidad, accesibilidad, equidad y calidad de los servicios de salud, enmarcadas, por un lado, en los objetivos trazados en la política de descentralización de los servicios de salud en México, y por el otro, teniendo en cuenta los estándares de salud planteados por la Organización Mundial de la Salud (OMS) y la Organización Panamericana de la Salud (OPS).

Resultados La política de descentralización de la salud en el Estado de Baja California desde 1996 y hasta el 2004 no ha logrado alcanzar el propósito central de esta política, en términos de sus objetivos sustantivos: disponibilidad, accesibilidad, equidad y calidad en la prestación de los servicios de salud.

Conclusiones En Baja California la población asegurada cuenta con más recursos que la población no asegurada, un factor que sin duda alguna influye para que no se de en la prestación de los servicios de salud la equidad, disponibilidad, acceso y calidad.

Palabras Clave: Descentralización, accesibilidad a los servicios de salud, equidad en salud, sistemas de salud, pacientes no asegurados (fuente: DeCS, BIREME).

\section{ABSTRACT}

Objective Documenting, defining and examining the state of Baja California's performance in decentralising public health service policy from the signing of the transfer agreement in 1996 up to 2004.

Methods This was a comparative study based on secondary information from 19962004. Four key categories were analysed: availability, accessibility, equity and health service quality. These categories formed part of the main goals in decentralising health service policy in Mexico and also took the health standards set by the World Health Organization and the Pan American Health Organization into account. 
REVISTADE SALUD PÚBLICA• Volumen 9 (4), Diciembre 2007

\begin{abstract}
Results The results revealed that decentralising health policy in Baja California from 1996-2004 had not ensured this policy's core objectives: availability, accessibility, equity, and health service quality.

Conclusions The population in Baja California having medical insurance had more resources than that part of the population lacking it, a factor without doubt influencing health services not providing equity, availability, accessibility and quality.
\end{abstract}

Key Words: health service accessibility, health system, patients lacking medical insurance (source: $M e S H, N L M$ ).

$\mathrm{E}$ l objetivo de conocer en forma específica la actuación del gobierno estatal obedece a que un proceso de esta magnitud conduce a una serie de reacomodos en el ámbito administrativo, político y social, que sin duda alguna ameritan ser conocidos de cerca para analizar los efectos que pueden originar las nuevas decisiones.

Teniendo en cuenta lo anterior, y analizando algunos estudios realizados, de diversas experiencias de descentralización en diversos países se puede ver en ellos cómo la descentralización se ha implementado con distintos niveles de profundidad e intensidad según el país que se analice. También ha tenido diferentes impactos en los gobiernos locales, en la institucionalidad regional o municipal, en la provisión de servicios, en la participación de la comunidad y, lo que es más importante, en las nuevas relaciones que se establecen a partir de estas reformas (1-6).

De ahí que, teniendo en cuenta los antecedentes del proceso de descentralización de los servicios de salud en México, los decretos y acuerdos firmados, tanto por el gobierno federal como estatal, y las diversas investigaciones orientadas a conocer los impactos de la política de descentralización en la vida nacional e internacional, esta investigación se encaminó a analizar, hasta donde la política de descentralización de los servicios de salud en Baja California, ha logrado el propósito central de esta política planteado desde el ámbito federal en términos de sus objetivos centrales: disponibilidad, accesibilidad, equidad y calidad en la prestación de éstos servicios, en especial a la población denominada abierta, es decir todas aquellas personas que carecen de un seguro de salud.

\title{
MATERIALES Y MÉTODOS
}

Con el fin de lograr el objetivo trazado hemos escogido para nuestro estudio el Instituto de Servicios de Salud Pública del estado de Baja California 
(ISESALUD), este a la vez incluye las jurisdicciones sanitarias de Mexicali, Tijuana y Ensenada. Las jurisdicciones Sanitarias se encuentran ubicadas en áreas geográficas determinadas, para ello se tiene en cuenta criterios como el número de habitantes, capacidad hospitalaria, acceso vial, etc. y son consideradas como oficinas representativas del propio ISESALUD en cada municipio. Es necesario aclarar que en esta investigación sólo se analiza el impacto de la descentralización en la población abierta, o no asegurada, la población derechohabiente o asegurada, se tendrá en cuenta solamente como un referente de comparación.

La investigación utilizó como método de recolección la revisión documental de fuentes secundarias que incluyen: i) documentación relacionada con el incremento de los recursos humanos: número de médicos, odontólogos, enfermeras, promotores, etc., en relación con el indicador sectorial en el país; ii) documentación concerniente al incremento de los recursos físicos y materiales: número de hospitales, centros de salud, ambulancias, camas, etc., comparada con el indicador sectorial en el ámbito nacional, y iii) documentación de los reportes presupuestarios y el financiamiento de la salud en los órdenes federal y estatal. Aunado a esto se analizaron los planes sectoriales de salud correspondientes a los dos gobiernos que les ha correspondido implementar la descentralización del sector salud en Baja California.

Categorías principales de análisis

Las categorías disponibilidad, accesibilidad, equidad y calidad utilizadas en esta investigación se enmarcan, por un lado, en los objetivos trazados en la política de descentralización de los servicios de salud en México y, por el otro, en los estándares de salud planteados por la Organización Mundial de la Salud (OMS) y por la Organización Panamericana de la Salud (OPS).

Estas categorías serán el termómetro para medir lo que ha representado la política de descentralización de los servicios de salud para Baja California, con el fin de observar de cerca qué factores han impactado de forma directa en la disponibilidad, la accesibilidad, la equidad y la calidad de los servicios de salud en el estado. Los aspectos que se tuvieron en cuenta para analizar estas categorías se indican a continuación.

Equidad y disponibilidad: Se analizaron desde el punto de vista de la distribución y disposición de recursos físicos y materiales (instalaciones, bienes y servicios, número de hospitales, clínicas, etc.), recursos humanos (existencia 
REVISTADE SALUD PÚBLICA•Volumen 9 (4), Diciembre 2007

de personal profesional y capacitado para desempeñar las funciones requeridas) y presupuestarios (destinación de presupuestos para la atención de la población) existentes para la prestación de los servicios de salud a la población abierta. En este análisis de las variables equidad y disponibilidad se realizo una comparación entre los recursos físicos y materiales, humanos y presupuestarios con los que cuenta la población no asegurada, frente a los de la población asegurada en los ámbitos estatal y nacional, entre los años 1996 y 2004, con el fin de dar cuenta si la política de descentralización de la salud de alguna manera ha logrado los objetivos sustantivos trazados para la población sin afiliación a la seguridad social (7-8).

Accesibilidad: En este estudio, para evaluar la accesibilidad se tuvo en cuenta la cantidad de población que ha asistido a recibir servicios de salud de los siguientes programas: planificación familiar, Diabetes Mellitus, hipertensión arterial, consultas especializadas y odontológicas (9-10).

Calidad: Esta variable tuvo como indicadores centrales para analizar los siguientes: atención a los usuarios que involucra tiempo de espera en consulta externa y urgencias, el tiempo de espera en consulta externa se define como la suma de los minutos que pasaron desde la asignación de la consulta hasta su entrada al consultorio médico, y el tiempo de espera promedio en urgencias es la suma de los minutos pasados por los usuarios desde que solicitan atención de urgencia hasta que ésta se inicia. Trato de calidad en unidades de primer nivel y urgencias. El desempeño en trato de calidad en unidades de primer nivel se evalúa a través de un índice que mide el tiempo efectivo de espera, la satisfacción del paciente con la información proporcionada por el médico sobre su diagnóstico y sobre su tratamiento y el surtimiento de medicamentos, y la correspondiente satisfacción del usuario con estos elementos. En el servicio de urgencias se evalúa mediante un índice que mide el grado de satisfacción del usuario (paciente, familiar o acompañante) con la oportunidad en la atención, el intercambio de información entre el médico y el usuario y el trato brindado por el personal en la unidad médica en servicios de urgencias en hospitales de áreas urbanas y rurales (11-14).

Análisis

El estudio incluye el análisis de datos para dos periodos de tiempo: años 1996 y 2004. La selección de estos dos periodos de estudio obedece fundamentalmente a dos razones: En el año 1996 el estado de Baja California se incorpora al proceso de descentralización del sector salud, con la firma del Acuerdo de Coordinación para la Descentralización Integral de los Servicios de Salud. El 
año 2004, año de corte de esta investigación. Se analizarán las acciones que han realizado los gobiernos del estado de Baja California a partir de la firma del convenio, para ver qué avances en materia de salud ha tenido la entidad en estos ocho años.

Estos dos periodos ofrecen la oportunidad de analizar los cambios que se produjeron en las variables de equidad, disponibilidad, accesibilidad y calidad de los servicios de salud en el estado. El primero (año 1996), nos da la oportunidad de conocer el estado general del sector salud en cuanto a recursos técnicos, materiales, humanos y presupuestarios, tiempo donde era responsable el nivel federal a través de la Secretaría de Salud. El segundo periodo (año 2004), ocho años después de la firma del convenio de transferencia, nos permite analizar a través de las estadísticas existentes y los planes sectoriales de salud, si la política de descentralización de la salud en Baja California implementada por los gobiernos estatales ha favorecido de alguna manera a la población que no cuenta con seguridad social, y si estas acciones han sido las suficientes para mantener o mejorar las condiciones de salud que predominaban en 1996 cuando el gobierno de la federación hizo entrega del manejo del sector salud al gobierno estatal.

\section{RESULTADOS}

Teniendo en cuenta las variables equidad y disponibilidad en cuanto a los recursos físicos y materiales, entre la población no asegurada y asegurada, se puede ver cómo en estos recursos la población asegurada cuenta con indicadores mayores frente a la no asegurada (Tabla 1). Pese a que la población asegurada es menor que la población no asegurada, los recursos físicos y materiales de los que dispone la segunda siguen siendo insuficientes para su atención. Un ejemplo de ello son las camas censables. Si se observan las columnas correspondientes a 1996, se puede apreciar cómo en cuanto a la disponibilidad la población asegurada contaba con 1,00 camas por cada mil habitantes, frente a 0,36 camas para la población abierta, es decir, más del doble. Lo mismo sucede con los demás recursos, como se observa en la misma tabla para los años 1996 y 2004.

Ahora bien, si se comparan los resultados de todo el estado para la población abierta y asegurada con los resultados a nivel nacional para ambas poblaciones, se puede advertir claramente que en cuanto a los recursos físicos y materiales Baja California está por debajo de la tasa nacional. Un ejemplo de ello son las unidades de consulta externa y hospitalización: mientras que para 1996 la tasa 
REVISTADE SALUD PÚBLICA· Volumen 9 (4), Diciembre 2007

nacional fue de 0,35 unidades por cada mil habitantes, en Baja California la tasa sólo fue de 0,17 unidades, y lo mismo sucedió para 2004, cuando la tasa nacional fue de 0,36 unidades por mil habitantes, para Baja California sólo fue de 0,16 unidades, una tasa menor incluso que la de 1996. Es decir, este recurso descendió para el estado pese a que la población había aumentado en un 35.8 $\%$.

Tabla 1. Comparativo de recursos físicos y materiales entre la población abierta y asegurada, Baja California, 1996-2004. Tasa X 1000 habitantes

\begin{tabular}{lcccc}
\hline Recursos Fisicos y materiales & \multicolumn{2}{c}{$\begin{array}{c}\text { Población No } \\
\text { asegurada }\end{array}$} & \multicolumn{3}{c}{$\begin{array}{c}\text { Población } \\
\text { asegurada }\end{array}$} \\
& $1996^{*}$ & $2004^{* *}$ & $1996^{*}$ & 2004* \\
\hline $\begin{array}{l}\text { U. de Consulta Extema y } \\
\text { hospitalización }\end{array}$ & 0,10 & 0,12 & 0,07 & 0,04 \\
Camas Censables & 0,36 & 0,43 & 1,00 & 0,69 \\
Camas no Censables & 0,29 & 0,27 & 0,42 & 0,34 \\
Consultorios & 0,31 & 0,40 & 0,46 & 0,37 \\
Incubadoras & 0,03 & 0,04 & 0,06 & 0,04 \\
Lab. de Análisis Clínicos y de & 0,01 & 0,01 & 0,02 & 0,02 \\
patologia & & & & \\
Gabinetes de Radiologia & 0,01 & 0,01 & 0,04 & 0,01 \\
Quirólanos & 0,02 & 0,01 & 0,04 & 0,03 \\
Salas de Expulsión & 0,04 & 0,03 & 0,03 & 0,02 \\
Bancos de Sangre & 0,00 & 0,00 & 0,01 & 0,01 \\
\hline Secretaría de Salud (1997); ** Instituto de Servicios de Salud Pública del Estado de Baja \\
California (ISESALUD) (2005).
\end{tabular}

Teniendo en cuenta las variables equidad y disponibilidad entre la población abierta y asegurada en cuanto a los recursos humanos, se puede percibir cómo los de la población no asegurada, y en algunos casos se triplican, como se estos recursos en la población asegurada cuenta con indicadores mayores que observa en el rubro de las enfermeras auxiliares, generales y especializadas (Tabla 2). Si se revisa la misma tabla tanto para 1996 como para 2004, se puede percibir que sucede algo parecido con los demás recursos.

Si consideramos que en la variable accesibilidad existen algunas condiciones indispensables para que todas las personas, por el derecho constitucional que les asiste, puedan tener y recibir del Estado atención para mejorar la salud, se puede decir que la población no asegurada de Baja California, frente a la asegurada, no ha recibido los beneficios que la ley y la política de descentralización del sector salud plantearon en su momento en cuanto a aumentar y facilitar el acceso de esta población a programas básicos de salud (Tabla 3). 
Tabla 2. Comparativo de recursos humanos, población abierta y asegurada, Baja California, 1996-2004. Tasa por mil habitantes

\begin{tabular}{lrrrr}
\hline \multicolumn{1}{c}{ Recursos humanos } & \multicolumn{2}{c}{$\begin{array}{c}\text { Población No } \\
\text { asegurada }\end{array}$} & \multicolumn{2}{c}{$\begin{array}{c}\text { Población } \\
\text { asegurada }\end{array}$} \\
\cline { 2 - 5 } & $1996^{*}$ & $2004^{*}$ & $1996^{*}$ & $204^{*}$ \\
\hline Enfermeras Auxiliares & 0,41 & 0,39 & 1,04 & 0,64 \\
Enfermeras Generales & 0,51 & 0,61 & 0,94 & 0,81 \\
Enfermeras Especializadas & 0,02 & 0,04 & 0,38 & 0,34 \\
Enfermeras en otras Labores & 0,18 & 0,21 & 0,06 & 0,1 \\
Otro Personal paramédico & 0,05 & 0,18 & 0,59 & 0,5 \\
Médicos Generales & 0,12 & 0,22 & 0,49 & 0,45 \\
Médicos Especialistas & 0,16 & 0,24 & 0,76 & 0,71 \\
Residentes & 0,04 & 0,14 & 0,02 & 0,04 \\
Pasantes & 0,10 & 0,14 & 0,01 & 0,02 \\
Odontólogos & 0,08 & 0,04 & 0,1 & 0,06 \\
En otras labores & 0,05 & 0,07 & 0,34 & 0,11 \\
Servicios Auxiliares de & 0,07 & 0,11 & 0,4 & 0,34 \\
Diagnóntico y Tratamiento & 0,38 & 0,42 & 0,78 & 0,4 \\
Personal Administrativo & & &
\end{tabular}

* Secretaría de Salud (1997); ** Instituto de Servicios de Salud Pública del Estado de Baja California (ISESALUD) (2005)

Tabla 3. Acceso a servicios básicos de salud de la población abierta y asegurada, Baja California, 1996-2004 expresado en porcentaje

\begin{tabular}{lrrrrr}
\hline \multirow{2}{*}{$\begin{array}{c}\text { Población adulta y adulta } \\
\text { mayor, hombres y mujeres }\end{array}$} & \multicolumn{2}{c}{$\begin{array}{c}\text { Población No } \\
\text { asegurada }\end{array}$} & \multicolumn{2}{c}{$\begin{array}{c}\text { Población } \\
\text { asegurada }\end{array}$} \\
\cline { 2 - 6 } & $1996^{*}$ & $2004^{* *}$ & $1996^{*}$ & $2004^{* *}$ \\
\hline Planificación familiar & 3,4 & 2,8 & 6,0 & 11,0 \\
Diabetes mellitus & $\mathrm{Nd}$ & 21,1 & $\mathrm{Nd}$ & 15,0 \\
Hipertensión arterial & $\mathrm{Nd}$ & 22,5 & $\mathrm{Nd}$ & 53,0 \\
Consultas especializadas & 7,0 & 13,9 & 54,0 & 45,0 \\
\hline Consultas odontológicas & 5,2 & 3,3 & 17,0 & 11,0 \\
\hline
\end{tabular}

*INEGI y Gobierno del Estado de Baja California (1997). Anuario Estadístico del Estado de Baja California; ** INEGI y Gobierno del Estado de Baja California (2005). Anuario Estadístico del Estado de Baja California; $\mathrm{Nd}=$ No disponible.

En la variable calidad, donde se evaluó los tiempos de espera en consulta externa y urgencias, se observa claramente cómo éstos en lugar de mejorar, es decir bajar, los minutos que los usuarios deben esperar para ser atendidos, al contrario aumentan cada año, lo que demuestra que el estado en cuanto a los servicios de salud se refiere ha bajado también en la calidad (Figura 1).

Dentro de los objetivos planteados en el proceso de descentralización de la salud en México, se encuentra el incremento del gasto público en salud a nivel federal y por estado, pero si analizamos el gasto público en salud de México como porcentaje del PIB en el año 2002, se puede ver que este fue de 6,1 \%, inferior al promedio de países latinoamericanos como Argentina, Brasil, Costa 
REVISTADE SALUD PÚBLICA·Volumen 9 (4), Diciembre 2007

Rica y Chile, de 7,8\%, y del promedio de los países de la misma OCDE -donde México forma parte desde hace 12 años-, que es de 7,9 \% (15).Ahora bien, si hacemos una comparación del gasto público en salud como porcentaje del PIB de 2001 a 2004 de Baja California con respecto al promedio nacional y a los dos primeros estados que presentan los PIB más altos del país, se puede ver cómo Baja California se encuentra en una condición muy desfavorable entre los 31 estados, incluido el Distrito Federal (Figura 2).

Figura 1. Tiempo de espera en consulta externa y urgencias Estado de Baja California 2001-2004

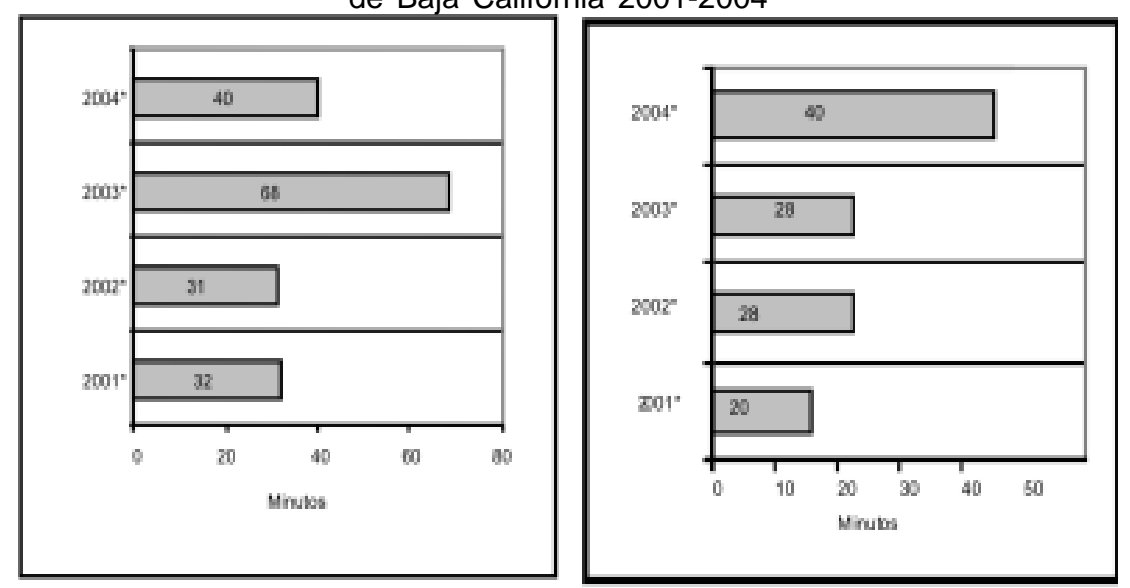

* Elaboración con base en los datos Salud: México 2001-2004. Información para Rendición de Cuentas, primera edición, Secretaría de Salud. México.

Figura 2. Gasto público en salud como porcentaje del Producto Interno Bruto$\mathrm{PIB}$, de Baja California con respecto al Promedio Nacional ya los dos Estados del País con el PIB más alto 2001- 2004

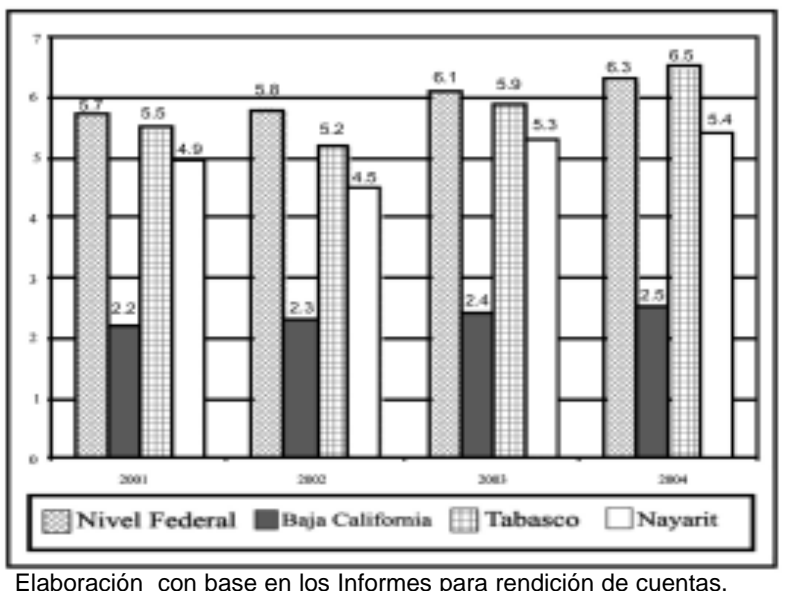

Elaboración con base en los Informes para rendición de cuentas,

Salud México 2001 al 2004. Secretaría de Salud. 


\section{DISCUSIÓN}

La mitad de la población mexicana está cubierta por la seguridad social y alrededor de la mitad del gasto en salud de las familias sin seguridad social proviene de los bolsillos de las personas, lo que genera un desmejoramiento en su calidad de vida, debido a que ese gasto no permite atender otras necesidades básicas, como alimento, educación, vivienda, etcétera. Estas cifras hacen ver que el país en general está lejos de lograr la cobertura universal o casi universal de la salud, una condición que también se vive en Baja California, donde en 2004 el 40 \% de la población no tenía seguridad social y la inversión del gobierno del estado para atenderla fue de sólo 0,5 \% del presupuesto general. En ese año, Baja California fue la entidad del país con la menor tasa de financiamiento para atender la salud de la población no asegurada (16).

Como se mostró en los resultados de esta investigación, en Baja California la población asegurada cuenta con más recursos que la población no asegurada, un factor que sin duda alguna influye para que la equidad, disponibilidad, acceso y calidad de los servicios de salud sean desventajosos para la población que no cuenta con seguridad social respecto de la asegurada. Estos resultados constituyen un factor más para considerar que la política de descentralización del sector salud en el estado no ha contribuido a mejorar las condiciones de salud de la población no asegurada. Las acciones emprendidas por el gobierno estatal entre 1996 y 2004 fueron insuficientes para lograr un impacto real de la política de descentralización del sector salud en la disponibilidad, equidad, acceso y calidad de los servicios, y esto obedeció básicamente a que las políticas públicas de salud fueron diseñadas atendiendo razones políticas, dejando de lado las necesidades reales que el estado tenía en salud.

La forma de gobernar en Baja California aún tiene sus matices tradicionales y centralizados, lo que en definitiva afecta el desarrollo de las políticas públicas, y más si los gobiernos no tienen capacidad para gestionar y negociar.

Finalmente, analizadas las estadísticas y contrastadas a la vez con los planes sectoriales de salud, se puede decir que: desde 1996 al 2004 la descentralización del sector salud ha tenido muy poca incidencia en el mejoramiento de los servicios públicos de salud en Baja California, siendo varios los aspectos que los gobiernos venideros deben replantearse para lograr los objetivos trazados de la descentralización, en donde sobresalen: la planeación a largo plazo y con una visión estratégica (donde se de continuidad a los planes y programas que se vienen desarrollando con los ajustes requeridos y donde esté presente la voluntad 
REVISTADE SALUD PÚBLICA• Volumen 9 (4), Diciembre 2007

de hacer eficiente al Sistema Estatal de Salud actual); la realización de procesos participativos (se debe incorporar los municipios y las organizaciones existentes, es decir tener una visión de la política desde las relaciones intergubernamentales) para diseñar las políticas de salud; la implantación de un sistema de evaluación propio que permita realizar los ajustes de forma oportuna (las iniciativas del gobierno federal para mejorar la salud pueden ser buenas y al igual el gobierno estatal puede participar de ellas, pero pese a ello, es urgente que el estado diseñe su propio sistema de evaluación) y finalmente, es necesario que las políticas de salud diseñadas e implementadas en todo el estado se hagan de forma focalizada, es necesario hacer un esfuerzo para generar un sistema que lleve a ubicar a la población no asegurada y poder así enfocar todos los esfuerzos a brindar la atención requerida a esta población

Agradecimientos. Especial agradecimiento al Colegio de la Frontera Norte (COLEF) por la beca otorgada para la realización de mis estudios doctorales, al Instituto de Servicios de Salud Pública del Estado de Baja California (ISESALUD) y a la Secretaría de Salud por todo el apoyo al facilitar la información requerida.

\section{REFERENCIAS}

1. Cabrero E. Las políticas descentralizadoras en el ámbito internacional. Análisis de tendencias y obstáculos en diversos países. Centro de investigación y docencia económica. México 1994; (19): 1-30.

2. Cabrero E. Análisis de experiencias nacionales de descentralización. En: Cabrero E. Las políticas descentralizadoras en México (1983-1993): Logros y desencantos. México: Miguel Ángel Porrúa; 1998.p.18-54.

3. Burki S, Guillermo EP, William D. Más allá del centro: la descentralización del Estado. Washington: Banco Mundial; 1999. p. 1-16.

4. Cardozo M. El ámbito sectorial. Análisis de la descentralización en el sector salud (1983-1993). En: Cabrero E. Las políticas descentralizadoras en México (19831993): Logros y desencantos. México: Miguel Ángel Porrúa; 1998.p. 189-274.

5. Ornelas C. La descentralización de los servicios de educación y salud en México. En: Di Gropello E. La descentralización de la educación y la salud: un análisis comparativo de la experiencia Latinoamericana. CEPAL; 1998.p. 187-207.

6. Rodríguez V. La política de centralización frente a las políticas de descentralización, 1970-1995. En: La descentralización en México: de la reforma municipal a la solidaridad y el nuevo federalismo. México: Fondo de Cultura Económica; 1997. p.133-179. 
7. Secretaría de Salud. Estadísticas recursos físicos materiales y humanos en las instituciones que proporcionan servicios médicos. México: Baja California; 1997.p. 1-22.

8. ISESALUD. Estadísticas principales recursos materiales de las unidades médicas en servicio de las instituciones públicas del sector salud según régimen e institución al 31 de diciembre de 2004. México: Baja California; 2005.p. 1-15.

9. INEGI. Gobierno del Estado de Baja California. Anuario estadístico del estado de Baja California. México: Aguascalientes; 1997.p. 225-283.

10. INEGI. Gobierno del Estado de Baja California. Anuario estadístico del estado de Baja California. México: Aguascalientes; 2005.p. 301-351.

11. Secretaria de Salud. Salud: México. Información para rendición de cuentas. México; 2001.p. 68-82.

12. Secretaria de Salud. Salud: México. Información para rendición de cuentas. México; 2002.p. 64-94.

13. Secretaria de Salud. Salud: México. Información para rendición de cuentas. México; 2003.p. 78-102.

14. Secretaria de Salud. Salud: México. Información para rendición de cuentas. México; 2004.p. 58-71.

15. OCDE. Estudios de la OCDE sobre los sistemas de salud: México. México: Secretaria de Salud; 2005. p. 19-76.

16. INEGI. XII Censo General de Población y Vivienda. México. 2000. [Internet] Disponible http://www.inegi.gob.mx/est/default.aspx?c=703 Consultado: 10 de junio de 2006. 